\title{
IMPACT OF A CORPULS CPR MECHANICAL CHEST COMPRESSION DEVICE ON CHEST COMPRESSION QUALITY DURING EXTENDED PAEDIATRIC MANIKIN RESUSCITATION: A RANDOMIZED CROSSOVER PILOT STUDY
}

\author{
Wojciech Wieczorek', Halla Kaminska² \\ ${ }^{1}$ Department of Anaesthesiology, Intensive Care and Emergency Medicine in Zabrze, Medical University of Silesia in Katowice, Poland \\ ${ }^{2}$ Department of Children's Diabetology, Medical University of Silesia, Katowice, Poland
}

\begin{abstract}
INTRODUCTION: The quality of chest compression delivered during paediatric cardiopulmonary resuscitation is identified as the most important factor to achieve the increase of the survival rate without a major neurological deficit to the patients. The aim of this study was to compare chest compression quality with and without the CORPULS CPR mechanical chest compression device during simulated paediatric cardiopulmonary resuscitation.
\end{abstract}

METHODS: A randomized crossover simulation trial was designed. 24 experienced paramedics participated in this trial. They performed paediatric chest compression with and without the CORPULS CPR chest compression device on a $\mathrm{HAL} \circledast \mathrm{S} 3005$ five year old paediatric simulator. They performed single-rescuer continuous chest compression in a 2-min scenario. The primary endpoint was compression depth.

RESULTS: The mean compression depth without CORPULS CPR was $4.7 \pm 0.2 \mathrm{~cm}$ and was statistically significant lower than when CORPULS CPR was used $7 \pm 0.3 \mathrm{~cm}$. The mean compression rate with and without CORPULS CPR was differentiated: $94 \pm 1$ vs. $100 \pm 5$.

CONCLUSIONS: This simulated scenario study showed that manual chest compression allows one to adjust the compression depth more precisely in comparison to CORPULS CPR device. The system compressed the simulator chest too deeply.

KEY WORDS: chest compression, quality, paramedic, cardiac arrest, paediatric

Disaster Emerg Med J 2017; 2(2): 58-63

\section{INTRODUCTION}

Lifesaving procedures performed on the paediatric population is one of the most difficult tasks in Emergency Medicine [1]. There is no doubt that this should be the major concern of public health and society. Life-threatening conditions in children play a key role in the psychological strain of medical workers. According to international reports, 17 million people die each year due to sudden cardiac arrest and for many patients this is the first manifestation of cardiovascular disease [2, 3]. The first few minutes after cardiac arrest are crucial for saving the patient's life [4]. Human body oxygen supplies will be lost and only appropriate and timely resuscitation 
is superior for patients experiencing cardiac arrest. High-quality chest compression, namely CPR provides critical perfusion to the heart and brain $[5,6]$.

In infants and children, cardiac arrest is less common than in adults [7-10]. In the paediatric population this results from progressive respiratory failure, shock or both. Less commonly, it can occur after injuries. According to these facts, life saving interventions in the paediatric population should be focused on enabling one to quickly recognize signs of respiratory distress, respiratory failure and shock. If not appropriately treated, cardiopulmonary failure and cardiac arrest can rapidly develop. It is understandable that in this population, high quality chest compression without open airway and ventilation might be insufficient.

According to international guidelines for CPR, high quality chest compressions are still fundamental. The universal chest compression rate is at least 100-120/min. During CPR provided by two rescuers, the compression-to-ventilation ratio is $15: 2[5,11]$. In most cases, chest compression can be done using one hand only or both. In the vast majority of children it is possible to use the same chest compression method as in the adult population: namely, both hands (place the heel of one hand on the other). In case of small children and infants, the one hand technique may help one to achieve the proper compression depth. High-quality CC for infants and neonatal is succeeded by pushing the lower third area of the sternum deeply and rapidly to the depth of $1 / 3$ of the chest anterior-posterior diameter (approximately $5 \mathrm{~cm})[11,12]$. The compression-to ventilation ratio (until advanced airway is placed) for a single rescuer is 30:2 in all groups (adults, infants and children). There is no evidence-based research which shows the use of the mechanical chest compression devices in paediatric population. It is taken into consideration that they may develop thorax injuries.

The aim of this study was to compare chest compression quality with and without the CORPULS CPR mechanical chest compression device during simulated paediatric cardiopulmonary resuscitation. The primary outcome was the compression depth. Secondary outcomes were the compression rate, the percentage of full release and the percentage of no flow fraction.

\section{METHODS}

This prospective, randomized, crossover study design was approved by the Institutional Review Board of the Polish Society of Disaster Medicine (Approval no.: 23.04.2017.IRB). 24 paramedics employed in the Emergency Medical Services in Poland were enrolled. All of them were well-experienced in emergency medicine and one year before the study had undergone a BLS course based on 2015 European Resuscitation Council CPR Guidelines. Before the project, informed consent was obtained from all of the participants.

\section{Device}

The CORPULS CPR device is a new-generation portable system which allow fully automated chest compression. The system's weight is $2.2 \mathrm{~kg}$ while its construction offers easy access to the patient's thorax from any side (Fig. 1). The system works on an electric power source. The operating time on an integrated battery typically takes 90-120 minutes. The system displays the remaining run time in minutes. In cases of an uncharged battery, it is possible to plug the CORPULS into an electrical network. The system offers the following compression parameters: a compression frequency 80 to 120 compressions/min (adjustable in increments of 1 compressions/min) and a compression depth: 2 to $6 \mathrm{~cm}$ (individually adjustable in increments of $0.1 \mathrm{~cm}$ ). There are no restrictions on the weight of the patient, while CORPULS CPR may be used on patients with a chest height from 14 to $34 \mathrm{~cm}$ and a chest width up to $48 \mathrm{~cm}$ (Fig. 2).

Furthermore, the system consists of "soft start" mode which allows one to adjust the proper strength of the compression. The first three chest compressions in a ratio 30:2 in each cycle, or in every 100 compressions in continuous mode are usually performed in $59-75 \%$ and $89 \%$ of power. This allows one to adjust the proper compression

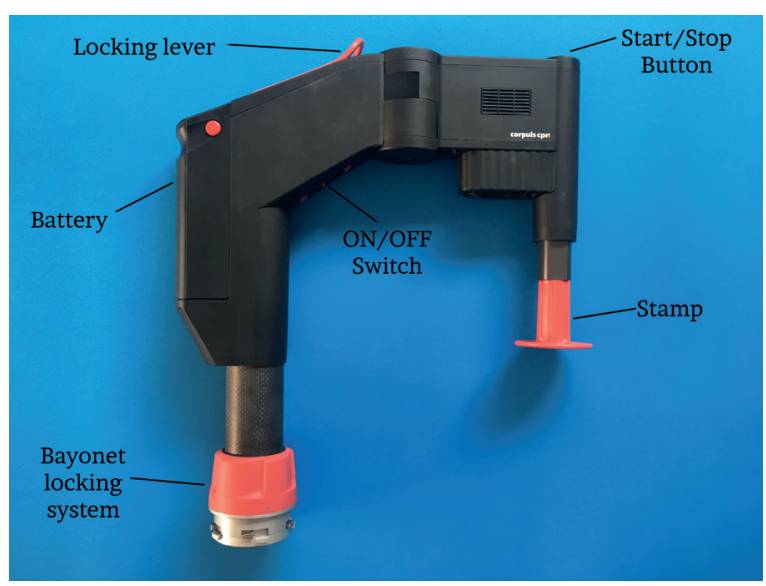

FIGURE 1. CORPULS CPR - general specifications 


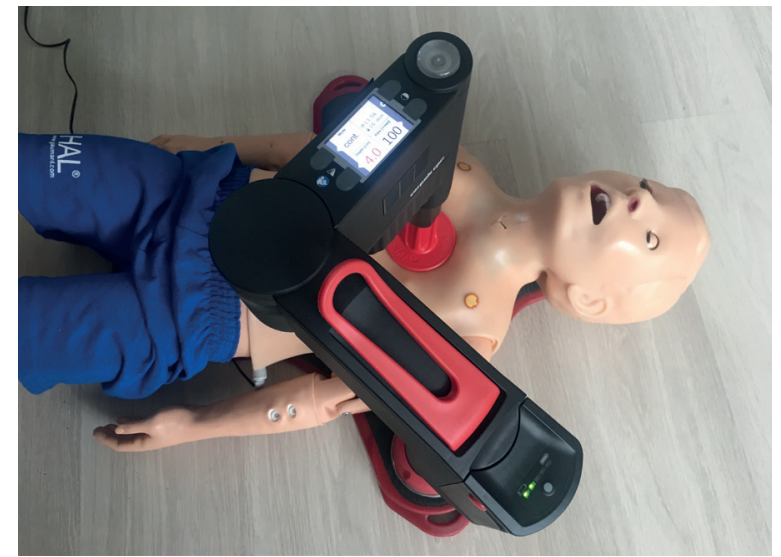

FIGURE 2. CORPULS CPR — during child's resuscitation

strength. CORPULS CPR makes it automatically. The CORPULS CPR checks the position of the stamp after each ventilation break or every 100 compressions (continuous mode), and automatically corrects the compression strength which compensates a sunken thorax (correction up to $0.8 \mathrm{~cm}$ ). The atraumatic power range is $270-620 \mathrm{~N}$ and is adjusted by a sensor.

\section{Simulation of a cardiac arrest patient}

A simulation-based cardiopulmonary resuscitation scenario was performed using a HAL ${ }^{\circledR}$ S3005 fiveyear-old paediatric simulator (Gaumard $\otimes$ Scientific, Miami, FL, USA). The mannequin was placed on a flat surface in a bright room.

\section{Intervention}

In a manual chest compression scenario, participants were told to perform single-rescuer continuous chest compression while kneeling beside the manikin at a rate and depth according to current European Resuscitation Council, as well as American Heart Association guidelines $[5,11]$. The participants were asked to compress the chest using the one-hand-only technique (Fig. 3). In the mechanical chest compression scenario, the subjects prepared and turned on the CORPULS CPR device. The system was programmed into a continuous chest compression mode with compression depth at $4 \mathrm{~cm}$ and a compression rate 100 per minute (Fig. 4). The study was designed as randomized controlled crossover. Both the order of the subjects and chest compression method were randomly allocated. The allocation ratio was $1: 1$. In each simulation scenario, CPR was performed by a single rescuer in 2 minutes. After one procedure, the participant was asked to relax in a 30-minute break, following which the

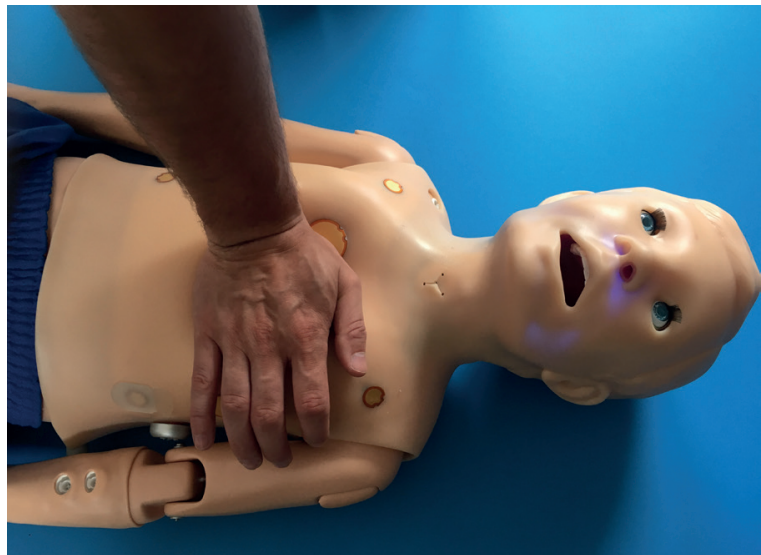

FIGURE 3. One hand chest compression method

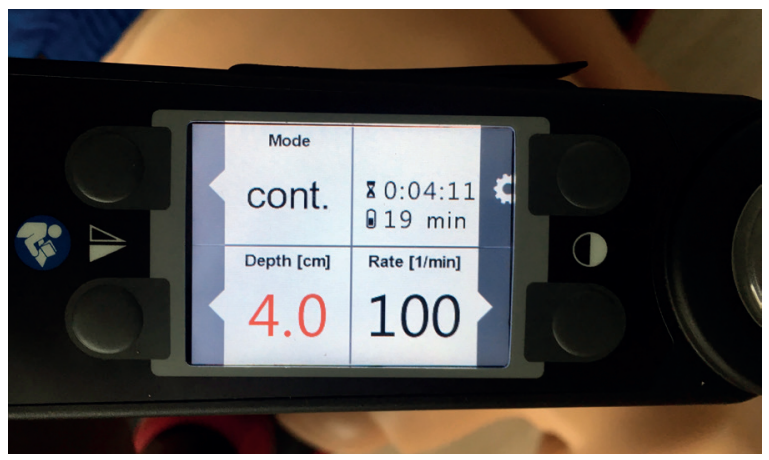

FIGURE 4. Control panel CORPULS CPR set according to study design

subject performed CPR using a different method. Figure 5 shows the detailed randomization process.

\section{Endpoints}

The primary outcome measure was the chest-compression depth. The secondary outcome measures chest compression depth, the percentage of full release and the percentage of no flow fraction. All the parameters were recorded using Control Pediatric $H A L \AA$ monitor software (Fig. 6), compatible with a simulator (Gaumard $\AA$ Scientific, Miami, FL, USA). Moreover, in order to collect, re-analyze and save them in the self-designed spreadsheet, all data were recorded using a GoPro HERO 5 Black (GoPRo Inc, San Mateo, CA, USA) camera.

\section{Statistical analysis}

All statistical analyses were performed using Statistica for Windows, Version 12.0 (StatSoft, Tulsa, USA). The Shapiro-Wilks test and examination of frequency histograms were used to analyze insertion time data. For data not normally distributed, the Wilcoxon signed rank test was used for non-parametric data. Descriptive values of variables are expressed 


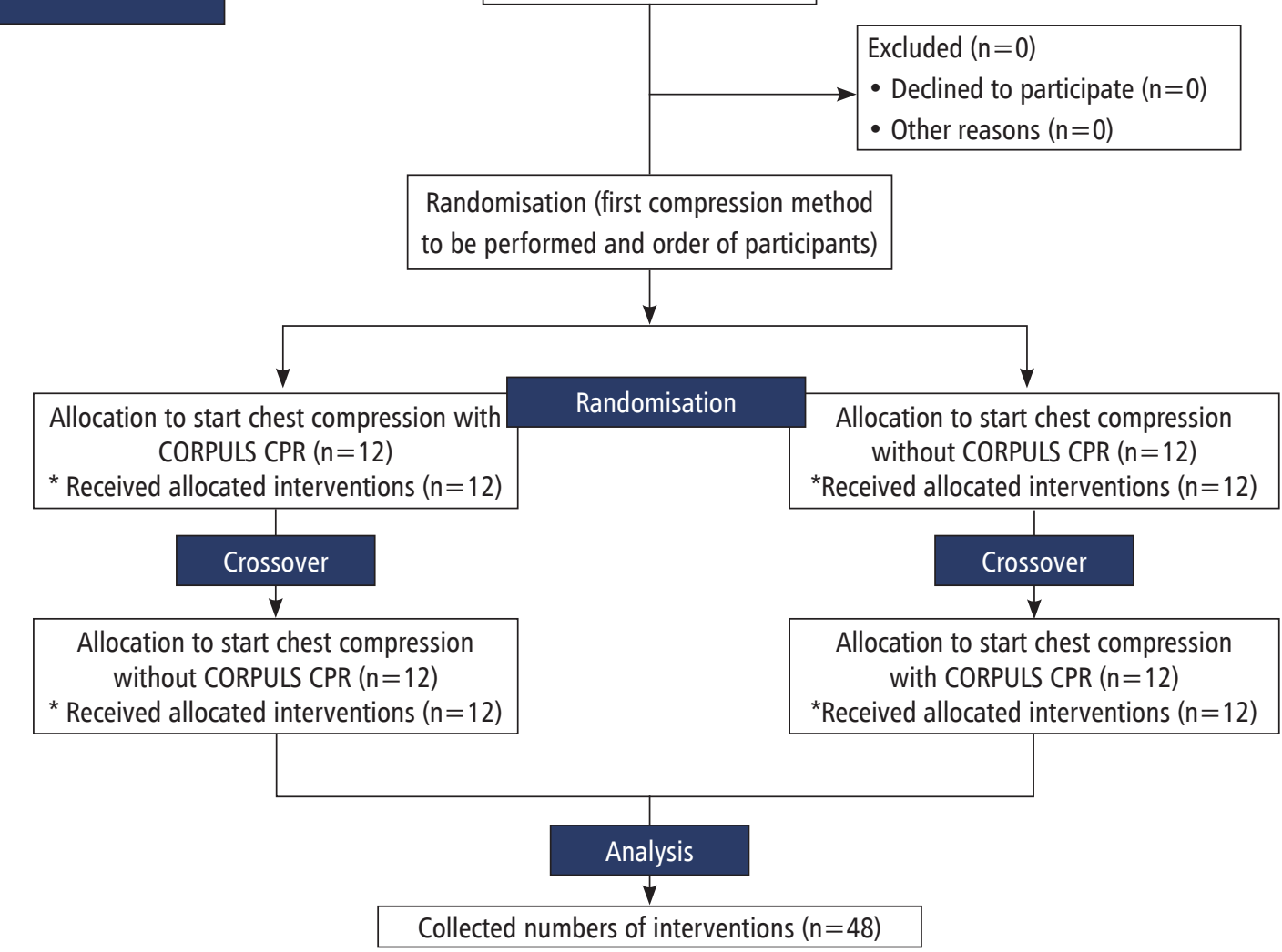

FIGURE 5. Randomization flow chart

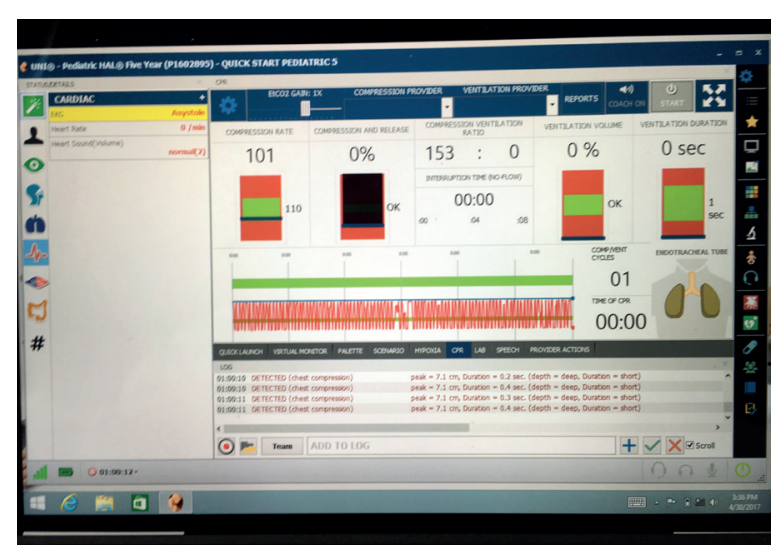

FIGURE 6. Control Pediatric HAL ${ }^{\circledR}$ monitor

as averages and percentages. In addition, p-Values were considered statistically significant when $\mathrm{p}<0.05$.

\section{Participants' characteristics}

24 paramedics (9 female; $37.5 \%$ ) participated in this study. The mean age was $34.5 \pm 9.5$ years while mean work experience was $10.2 \pm 4.5$ years. All participants worked in the Emergency Medical Services in Poland and during the previous year had undergone an updated BLS course according to ERC Guidelines.

\section{End points}

The mean compression depth without CORPULS CPR was $4.7( \pm 0.2 \mathrm{~cm})$ and was statistically significant lower than when CORPULS CPR was used $(7 \pm 0.3 \mathrm{~cm} ; \mathrm{p}<0.001)$. The mean chest compression with and without CORPULS CPR was diversified: $94 \pm 1$ vs. $100 \pm 5(p=0.044)$. No flow fraction in case of using the manual chest compression was lower in comparison to the CORPULS CPR system (Tab. 1).

\section{DISCUSSION}

European Resuscitation Council and American Heart Association guidelines recommend and support the importance of high quality chest compressions during $\operatorname{CPR}[5,11]$. 
Table 1. Study outcome measure measured variables of both groups presented as mean value \pm standard deviation

\begin{tabular}{|l|c|c|c|}
\hline \multicolumn{1}{|c|}{ Parameters } & With CORPULS CPR & Without CORPULS CPR & p-value \\
\hline Compression depth $[\mathrm{cm}]$ & $7 \pm 0.3$ & $4.7 \pm 0.2$ & $<0.001$ \\
\hline Compression rate $\left[/ \mathrm{min}^{-1}\right]$ & $94 \pm 1$ & $100 \pm 5$ & 0.044 \\
\hline Full release (\%) & $93 \pm 3$ & $81 \pm 8$ & 0.045 \\
\hline No flow fraction (\%) & $2 \pm 1$ & $0 \pm 0$ & $<0.001$ \\
\hline
\end{tabular}

A high quality of chest compression performance (hand placement, depth, rate, chest recoil, ventilation volume, compression interruption time, compression: ventilation ratio, and breathing intervals) during resuscitation establishes a functional residual capacity without causing lung injury and optimizing oxygen delivery to the tissues, optimizes blood flow to vital organs and achieves the return of spontaneous circulation $[13,14]$.

According to the updated guidelines from European and Resuscitation Council, high-quality CC for infants and neonatal patients is achieved by pushing the lower third area of the sternum deeply and rapidly to the depth of $1 / 3$ of the chest anterior-posterior diameter (approximately $5 \mathrm{~cm}$ ). For both, the compression rate should be at least 100 but not greater than $120 \mathrm{~min}$ with the minimization of interruptions and avoidance of excessive ventilation. Apart from that mentioned above an optimal and efficient way of treatment during CPR is endotracheal intubation and the implementation of continuous chest compression - in order to minimize interruptions in chest compression.

Our study showed that high quality cardiopulmonary resuscitation of a 5 -year-old child provided by well-experienced paramedics demonstrated a mean compression depth of $4.7 \mathrm{~cm}$. Moreover, simulator used in the study, in which the chest height was $14.5 \mathrm{~cm}$, the compression depth performed by study participants was equal to ERC and AHA guidelines $[4,5,13]$. CORPULS CPR was programmed to perform $4 \mathrm{~cm}$ compression depth. Nevertheless, the CORPULS CPR device showed deeper compressions than it is recommended for paediatric patients. The mean compression depth in this case was $7.0 \mathrm{~cm}$. Both ERC and AHA guidelines for children recommend a compression depth $1 / 3$ of the chest anterior-posterior diameter, while in adults this is $5-6 \mathrm{~cm}$. Results obtained after using CORPULS CPR exceeded the recommended ranges of chest compression depth. A possible complication may be organ damage and fracture of thorax scaffold. Steill et al. indicated a strong association between survival outcomes and increased compression depth but no clear evidence to support or refute the 2010 recommendations of $>50 \mathrm{~mm}$. Although compression depth is an important component of CPR and should be measured routinely, the most effective depth is currently unknown [15]. Other hand, Edelson et al. in large study of in-hospital cardiac arrest debriefing, demonstrated better ROSC with better overall CPR performance but did not isolate specific compression depth levels as a factor [16]. However, the above-mentioned studies were performed on an adult population, where the chest height is higher and the compression depth should be deeper in comparison to the paediatric population.

Our trial has several limitations. Firstly, the study was conducted in a simulation centre which is different from a real-life resuscitation situation. Notwithstanding it is ethically questionable to perform randomized, crossover studies during life-threatening situations. It is worth noting that simulation-based trials are universal and allow one to standardise research without the risking patient's health and life. Secondly, although the subjects enrolled to the study were only paramedics, the choice of this study group was deliberate. Working in the Emergency Medical Service exposes paramedics to the necessity of performing resuscitation on either a child or an adult.

The strength of this research is the use of a high quality and technologically advanced paediatric simulator, along with the crossover design of the study.

\section{CONCLUSIONS}

This simulated scenario study showed that the manual chest compression allows one to adjust the compression depth more precisely in comparison to the CORPULS CPR device. The system compressed the simulator chest too deeply.

Conflict of interest: None declared. 


\section{REFERENCES}

1. Ali S, Thomson D, Graham TAD, et al. High stakes and high emotions: providing safe care in Canadian emergency departments. Open Access Emerg Med. 2017; 9: 23-26, doi: 10.2147/0AEM.S122646, indexed in Pubmed: 28176924.

2. Orkin AM, Zhan C, Buick JE, et al. Out-of-hospital cardiac arrest survival in drug-related versus cardiac causes in Ontario: A retrospective cohort study. PLoS One. 2017; 12(4): e0176441, doi: 10.1371/journal. pone.0176441, indexed in Pubmed: 28445501.

3. Requena-Morales R, Palazón-Bru A, Rizo-Baeza MM, et al. Mortality after out-of-hospital cardiac arrest in a Spanish Region. PLoS One. 2017; 12(4): e0175818, doi: 10.1371/journal.pone.0175818, indexed in Pubmed: 28407020.

4. Singletary EM, Charlton NP, Epstein JL, et al. Part 15: First Aid: 2015 American Heart Association and American Red Cross Guidelines Update for First Aid. Circulation. 2015; 132 (18 Suppl 2): S574-S589, doi: 10.1161/CIR.0000000000000269, indexed in Pubmed: 26473003.

5. Maconochie IK, Bingham R, Eich C, et al. Paediatric life support section Collaborators. European Resuscitation Council Guidelines for Resuscitation 2015: Section 6. Paediatric life support. Resuscitation. 2015; 95: 223-248, doi: 10.1016/j.resuscitation.2015.07.028, indexed in Pubmed: 26477414.

6. Zhan L, Yang LiJ, Huang Yu, et al. Continuous chest compression versus interrupted chest compression for cardiopulmonary resuscitation of non-asphyxial out-of-hospital cardiac arrest. Cochrane Database Syst Rev. 2017; 3: CD010134, doi: 10.1002/14651858.CD010134.pub2, indexed in Pubmed: 28349529.

7. Christensen RE, Haydar B, Voepel-Lewis TD. Pediatric Cardiopulmonary Arrest in the Postanesthesia Care Unit, Rare but Preventable: Analysis of Data From Wake Up Safe, The Pediatric Anesthesia Quality Improvement Initiative. Anesth Analg. 2017; 124(4): 1231-1236, doi: 10.1213/ANE.0000000000001744, indexed in Pubmed: 28166099.

8. Deakin $C D$, England $S$, Diffey $D$, et al. Can ambulance telephone triage using NHS Pathways accurately identify paediatric cardiac arrest? Resuscitation. 2017 [Epub ahead of print], doi: 10.1016/j. resuscitation.2017.03.013, indexed in Pubmed: 28315728.
9. Risgaard B. Sudden cardiac death: a nationwide cohort study among the young. Dan Med J. 2016; 63(12), indexed in Pubmed: 27910804.

10. Stephens $P$. Sudden cardiac death in the young: the value of exercise testing. Cardiol Young. 2017; 27(S1): S10-S18, doi: 10.1017/ S1047951116002171, indexed in Pubmed: 28084955.

11. Atkins DL, Berger S, Duff JP, et al. Part 11: Pediatric Basic Life Support and Cardiopulmonary Resuscitation Quality: 2015 American Heart Association Guidelines Update for Cardiopulmonary Resuscitation and Emergency Cardiovascular Care. Circulation. 2015; 132(18 Suppl 2): S519-S525, doi: 10.1161/CIR.0000000000000265, indexed in Pubmed: 26472999.

12. de Caen AR, Berg MD, Chameides L, et al. Part 12: Pediatric Advanced Life Support: 2015 American Heart Association Guidelines Update for Cardiopulmonary Resuscitation and Emergency Cardiovascular Care. Circulation. 2015; 132(18 Suppl 2): S526-S542, doi: 10.1161/ CIR.0000000000000266, indexed in Pubmed: 26473000.

13. Perkins GD, Handley AJ, Koster RW, et al. Adult basic life support and automated external defibrillation section Collaborators. European Resuscitation Council Guidelines for Resuscitation 2015: Section 2. Adult basic life support and automated external defibrillation. Resuscitation. 2015; 95: 81-99, doi: 10.1016/j.resuscitation.2015.07.015, indexed in Pubmed: 26477420.

14. Lim JS, Cho Y, Ryu S, et al. Comparison of overlapping (OP) and adjacent thumb positions (AP) for cardiac compressions using the encircling method in infants. Emerg Med J. 2013; 30(2): 139-142, doi: 10.1136/emermed-2011-200978, indexed in Pubmed: 22402909.

15. Stiell IG, Brown SP, Christenson J, et al. Resuscitation Outcomes Consortium (ROC) Investigators. What is the role of chest compression depth during out-of-hospital cardiac arrest resuscitation?. Crit Care Med. 2012; 40(4): 1192-1198, doi: 10.1097/CCM.0b013e31823bc8bb, indexed in Pubmed: 22202708.

16. Edelson DP, Litzinger $B$, Arora $V$, et al. Improving in-hospital cardiac arrest process and outcomes with performance debriefing. Arch Intern Med. 2008; 168(10): 1063-1069, doi: 10.1001/archinte.168.10.1063, indexed in Pubmed: 18504334. 